\title{
Molecular Tension Probes to Quantify Cell-Generated Mechanical Forces
}

\author{
Kyung Yup Baek ${ }^{1,2}$, Seohyun Kim ${ }^{1,2}$, and Hye Ran Koh ${ }^{1, *}$
}

${ }^{1}$ Department of Chemistry, Chung-Ang University, Seoul 06974, Korea, ${ }^{2}$ These authors contributed equally to this work. *Correspondence: hrkoh@cau.ac.kr https://doi.org/10.14348/molcells.2022.2049

www.molcells.org

\begin{abstract}
Living cells generate, sense, and respond to mechanical forces through their interaction with neighboring cells or extracellular matrix, thereby regulating diverse cellular processes such as growth, motility, differentiation, and immune responses. Dysregulation of mechanosensitive signaling pathways is found associated with the development and progression of various diseases such as cancer. Yet, little is known about the mechanisms behind mechano-regulation, largely due to the limited availability of tools to study it at the molecular level. The recent development of molecular tension probes allows measurement of cellular forces exerted by single ligandreceptor interaction, which has helped in revealing the hitherto unknown mechanistic details of various mechanosensitive processes in living cells. Here, we provide an introductory overview of two methods based on molecular tension probes, tension gauge tether (TGT), and molecular tension fluorescence microscopy (MTFM). TGT utilizes the irreversible rupture of double-stranded DNA tether upon application of force in the piconewton $(\mathrm{pN})$ range, whereas MTFM utilizes the reversible extension of molecular springs such as polymer or single-stranded DNA hairpin under applied $\mathrm{pN}$ forces. Specifically, the underlying principle of how molecular tension probes measure cell-generated mechanical forces and their applications to mechanosensitive biological processes are described.
\end{abstract}

Keywords: cellular forces, mechanobiology, molecular spring, molecular tension fluorescence microscopy, tension gauge tether, tension probes

\section{INTRODUCTION}

Cells sense and generate mechanical forces as they interact with other cells or surrounding extracellular matrix (ECM) (Discher et al., 2005), in a process termed as mechanotransduction. These mechanical forces regulate diverse cellular processes such as cell adhesion (Parsons et al., 2010), migration (Kuo, 2013), proliferation (Hoffman et al., 2011; Uroz et al., 2018), differentiation (Engler et al., 2006), development (Vining and Mooney, 2017), immune response (Huse, 2017), and more (Jansen et al., 2015; Vogel and Sheetz, 2006). Defects in mechanotransduction often lead to the development and progression of diseases including skin disorder, muscular dystrophy, and cancer (Butcher et al., 2009; Levental et al., 2009). In mechanotransduction pathways, forces are either transmitted through a mechanosensitive receptor such as integrin by its direct interaction with a cognate ligand or through ECM by its physical deformation under tension, compression, or fluid shear stress (Fig. 1). The transmitted mechanical forces can either activate transcription of genes (Eroshkin and Zaraisky, 2017; Mammoto et al., 2012), recruit adaptor molecules for downstream cell signaling (Wang et al., 2007), induce actin polymerization at focal adhesions (Hirata et al., 2008), or activate mechanosensitive ion channels (Martinac, 2004) (Fig. 1). Even with such accumulated evidence indicating that cellular mechanical forces are important regulatory factors in diverse biological processes, little is known about the mechanism behind their regulation.

Quantification of cellular forces is a prerequisite to under-

Received 22 December, 2021; accepted 10 January, 2022; published online 26 January, 2022 

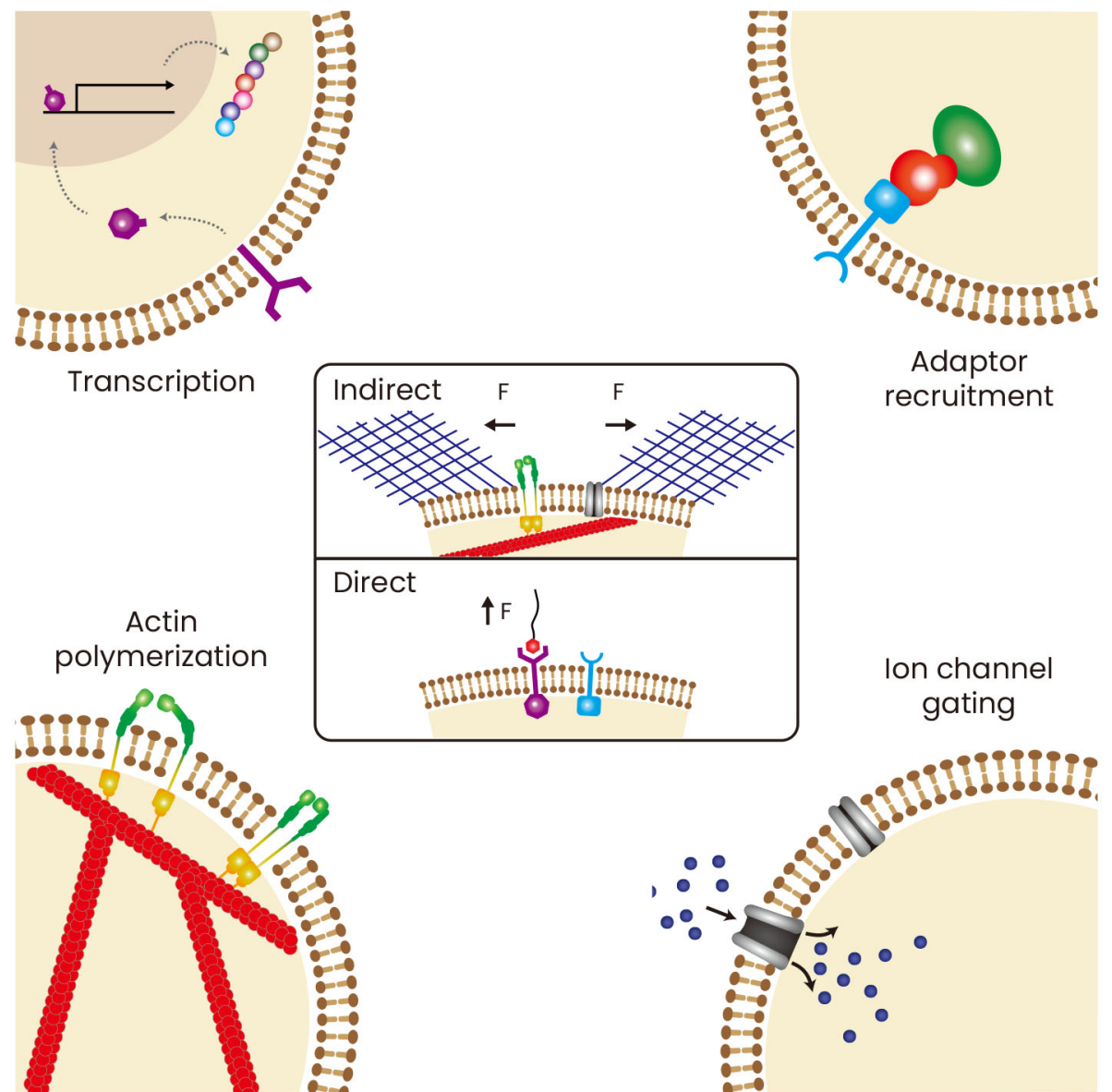

Fig. 1. Schematic of force-dependent cellular pathways. Cell senses mechanical forces generated by ligand-receptor interaction (direct) or by deformation of ECM (indirect) and responds to them either via gene transcription, adaptor recruitment, actin polymerization, or ion channel gating.

stand various mechanosensitive biological processes. The forces exerted by individual proteins and growing microtubules are in the range of piconewton (pN) (Dogterom and Yurke, 1997; Fisher and Kolomeisky, 1999), suggesting that sensitive techniques are required while investigating mechanotransduction at the molecular level. Various techniques to measure cell-generated mechanical forces have been developed (Pramanik, 2004; Roca-Cusachs et al., 2017). Traction force microscopy (TFM) measures the traction forces generated by distortion of adherent cells lying on a soft surface such as polyacrylamide gel (Harris et al., 1980; Roy et al., 2002; Style et al., 2014). As cells migrate over the soft surface, they get deformed by the cellular traction force, which can be measured by observing the degree of the deformation. However, the force sensitivity of TFM is at the nanonewton level, which is not enough to measure cellular forces applied by individual receptors or proteins. Single-molecule force spectroscopy (SMFS) (Neuman and Nagy, 2008) techniques such as atomic force microscopy (Gaub and Muller, 2017), optical tweezers (Riesenberg et al., 2020), and magnetic tweezers (Wang et al., 1993) exhibit improved sensitivity and can quantify cellular forces at the pN level. However, SMFS lacks high-throughput efficiency as it measures mechanical forces for one cell at a time. More recently, molecular tension probes are developed (Goktas and Blank, 2017; Liu et al., 2017; Ma and Salaita, 2019), which are high-throughput methods with high sensitivity to quantify cellular forces at the pN level.

In this mini-review, we focus on molecular tension probes and describe two molecular imaging methods based on such probes, tension gauge tether (TGT) and molecular tension fluorescence microscopy (MTFM). We also discuss the underlying principle of each method and its applications in understanding mechanosensitive biological processes in living cells.

\section{TGT}

In TGT, double-stranded DNA (dsDNA) conjugated with a ligand of interest is immobilized on a solid surface via biotinstreptavidin linkage (Fig. 2A). When cells are plated on the surface coated with the dsDNA, the cellular receptor interacts with the ligand conjugated to the dsDNA, exerting cellular 
A

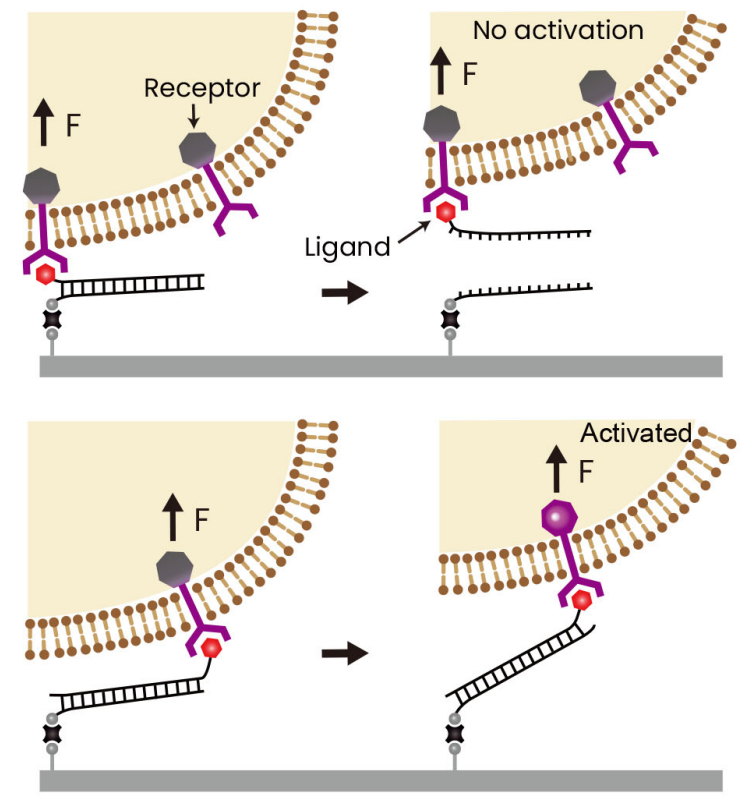

D

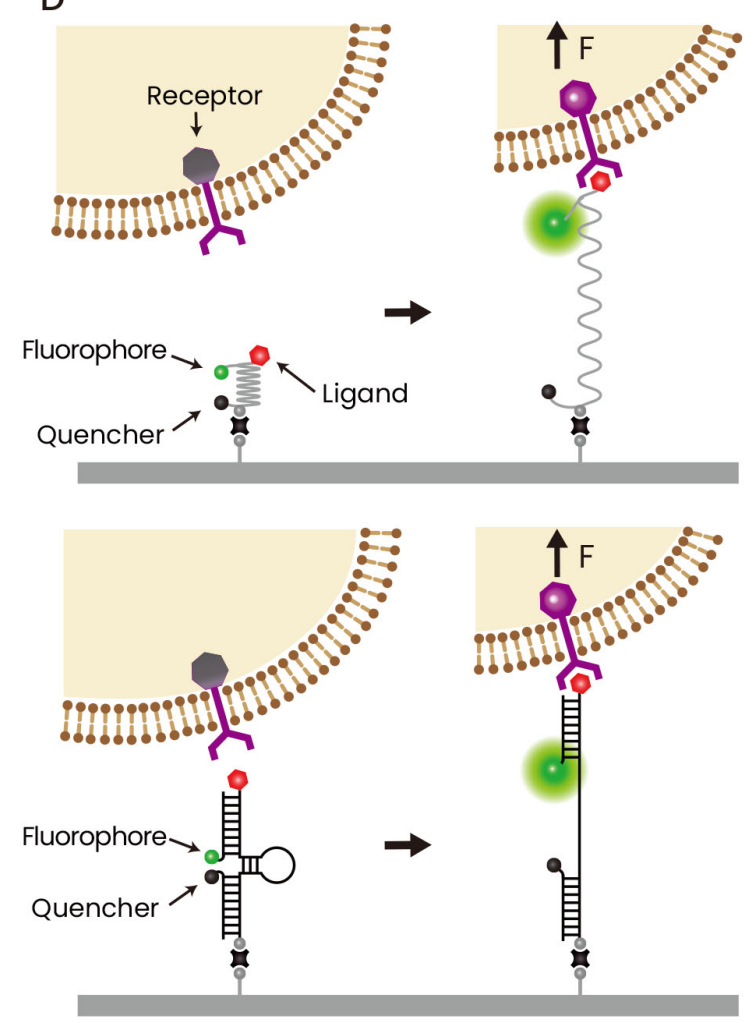

B

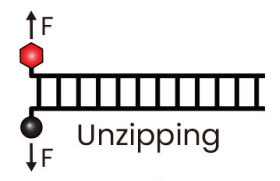

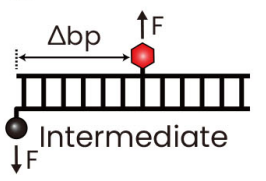

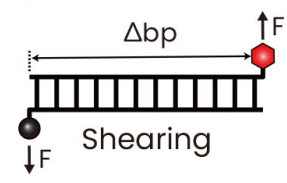

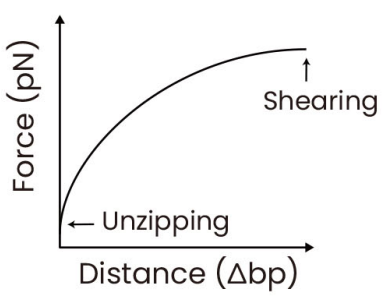

$\mathrm{E}$
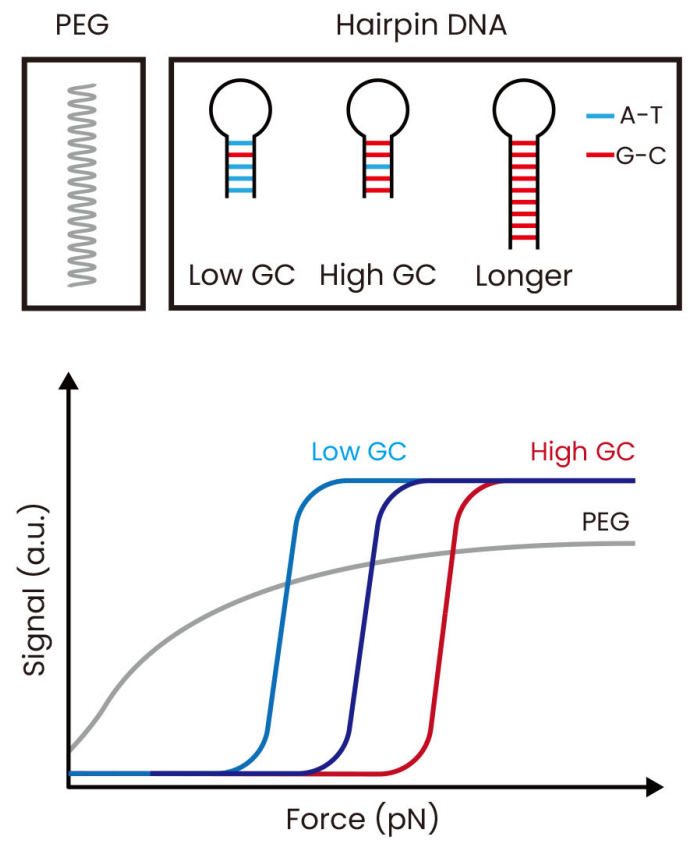

Fig. 2. Schematics of TGT and MTFM to quantify cellular forces at the pN level. (A) Schematic of TGT assay. The cellular force larger than the rupture force of the dsDNA tether activates the mechanosensitive receptor. (B) The rupture force of dsDNA depends on the force application geometry, which is the lowest at the unzipping geometry and the highest at the shearing geometry. (C) Signal of cell activation increases with increasing rupture force of dsDNA tether, exhibiting the threshold force required for cell activation marked by a dotted line. (D) Schematics of MTFM using PEG (top) and DNA hairpin (bottom) as a tension probe. A molecular tension probe is conjugated with a ligand of interest, a fluorophore, and a quencher. (E) DNA hairpin exhibit a more abrupt change of fluorescent intensity compared with PEG at the threshold force in response to external forces, and the percentage of GC content and the length of the DNA hairpin stem modulate the intensity of threshold force. 
mechanical forces. The dsDNA gets physically ruptured if the force exerted by the ligand-receptor interaction is larger than the rupture force of dsDNA, abolishing activation of the mechanosensitive receptor (Fig. 2A, top). On the contrary, if the force exerted by ligand-receptor interaction is smaller than the rupture force of dsDNA, dsDNA will endure, activating the receptor-mediated signaling (Fig. 2A, bottom).

Further, it was shown that dsDNA ruptures at a critical force, which largely depends on the geometry of force application (Albrecht et al., 2003). For instance, the critical force required for dsDNA rupture is lowest at the unzipping geometry, highest at the shearing geometry, and in between at the intermediate geometry (Fig. 2B). The correlation between the force application geometry and the applied force is well established, with rupture force of a 21-base pair dsDNA estimated as approximately $12 \mathrm{pN}$ in the unzipping geometry and approximately $56 \mathrm{pN}$ in the shearing geometry (Albrecht et al., 2003; Essevaz-Roulet et al., 1997; Wang and $\mathrm{Ha}$, 2013). Several dsDNA tethers with varying degrees of rupture force are examined to investigate the mechanical force required for activation of the mechanosensitive receptor. The cell signal would appear only if the cellular forces are well below the rupture force of the dsDNA tether, and an increase in the signal would be observed with an increase in rupture force of the dsDNA tether (Fig. 2C). Thus, the threshold force required for cell activation can be calculated by plotting the rupture force of dsDNA tether against the receptor signal as shown by the dotted line in Fig. 2C.

First-generation TGT probes monitor the signal of force-induced cell activation without revealing the rupture state of dsDNA tethers or the location of ligands (Wang and $\mathrm{Ha}$, 2013). A fluorescently labeled TGT developed in 2015, enabled the visualization of ligand distribution along with the measurement of force-induced cell activation (Wang et al., 2015). Unlike the earlier dsDNA tether design, in this TGT, a fluorophore is conjugated to one strand of dsDNA, which gets lost on the dsDNA rupture, thus providing the location of ligands in living cells through a fluorescent signal. If the force generated by ligand-receptor interaction is well above the rupture force of the dsDNA tether, the dsDNA gets ruptured and the tether fluorescence would disappear. Therefore, the distribution of forces exerted from each ligand-receptor interaction could be measured by fluorescence imaging. However, quenching of fluorophores can be misunderstood as the rupture of the dsDNA tether. To overcome this limitation, alternative methods such as integrative tension sensor (ITS) and quenched TGT (qTGT) were developed, where a fluorescent signal would appear only if dsDNA tether ruptured (Jo et al., 2019; Wang et al., 2018). In ITS and qTGT, a quencher and a fluorophore were conjugated to dsDNA tether, which helps in quenching the fluorescent signal in the absence of dsDNA rupture. To further improve the detection sensitivity of TGT probes, a mechanically induced catalytic amplification reaction was incorporated that produces fluorescently tagged amplicons after rupturing of dsDNA tether and thus amplifies the signal (Ma et al., 2016). Another limitation of TGT is its irreversible nature, where TGT probes cannot be used for multiple measurements. Recently, several tension probes to overcome this limitation were developed, which include a reversible shearing DNA probe (Li et al., 2021) and serially connected force sensors (Murad and Li, 2019).

\section{MTFM}

Some molecules possess the ability to extend just like springs when an external force is applied, and thus could serve as molecular tension probes (Clausen-Schaumann et al., 2000). These molecular springs include polymer, oligonucleotides, or proteins, exhibiting different force-extension relationships. In MTFM (Liu et al., 2017), along with a ligand of interest, a fluorophore and a quencher are conjugated to the molecular spring, which is immobilized on a solid surface via biotinstreptavidin linkage (Fig. 2D). When cells are plated on the surface, the cellular receptor interacts with its cognate ligand conjugated to the molecular tension probes, leading to the exertion of force and subsequent extension of the molecular spring. Initially, the fluorescence signal would be weak due to the proximity of the fluorophore to the quencher. However, as the cellular forces generated by ligand-receptor interaction extend the molecular spring, the distance between a fluorophore and a quencher increases, thereby increasing the fluorescent signal. Thus, fluorescent intensity directly depends on the magnitude of external force, making it possible to quantify the cellular forces by measuring the degree of extension in molecular tension probes. Molecular tension probes in MTFM differ from TGT probes in terms of their reversibility as they return to their original conformation when the applied force is removed.

The molecular spring used in the first-generation MTFM probes was a flexible polyethylene glycol (PEG) entropic spring conjugated with epidermal growth factor (EGF) ligand (Fig. 2D, top) (Stabley et al., 2012). PEG spring consisting of 24 monomer units and labeled with a fluorophore and a quencher exhibited a gradual increase in fluorescent intensity with a corresponding increase in applied force up to $20 \mathrm{pN}$ as PEG does not possess a defined secondary structure but a random coil conformation (Fig. 2E). From this positive correlation between force and fluorescent intensity, the cellular force could easily be quantified. Other molecular springs such as elastic polypeptides show a similar force-dependent extension with PEG (Morimatsu et al., 2013). For instance, elastic spider silk peptide consisting of (GPGGA), flanked by a fluorescence resonance energy transfer pair, was developed to quantify cellular forces in a range of 1-11 pN (Brenner et al., 2016: Morimatsu et al., 2013).

Next-generation MTFM probes employ DNA hairpins where single-stranded DNA (ssDNA) forms stem-loop structure (Fig. 2D, bottom) (Blakely et al., 2014; Zhang et al., 2014). On ligand-receptor interaction, the stem-loop structure of the DNA hairpin unfolds abruptly to assume ssDNA form. It works like the PEG-based MTFM probe, but fluorescent intensity sharply increases at a certain magnitude of the force that depends on the GC content and length of the stem in DNA hairpin (Fig. 2E). For instance, 100\% GC content in 12-bp stem of DNA hairpin exhibited rupture force of $19.3 \mathrm{pN}$ while $22 \%$ GC content in 9-bp stem showed only 4.7 pN of rupture force (Woodside et al., 2006; Zhang et al., 2014). The sharper increase in fluorescence intensity 
makes DNA hairpin more sensitive to measure cellular forces in comparison with PEG. Alpha-helical or beta-sheet polypeptides also behave like DNA hairpin since they also possess a well-defined secondary structure. For example, using an MTFM probe developed with the 27th immunoglobulin domain of cardiac titin (127) flanked with a fluorophore and a gold nanoparticle (AuNP), cellular forces could be measured in the range of 80-200 pN (Galior et al., 2016).

Most MTFM probes conjugated with a fluorophore and a quencher are immobilized on a solid surface via biotinstreptavidin linkage. Despite the strong interaction between biotin and streptavidin, integrin forces sometimes lead to the dissociation of the MTFM probe from the surface, interfering with the measurement of cellular forces (Jurchenko et al., 2014). AuNP provides a more stable linkage for surface immobilization through thiol-gold covalent bonding, reducing the dissociation probability of MTFM probes from the surface. AuNP can also serve as an efficient quencher, so both biotin-streptavidin linkage and quencher were replaced with AuNP to engineer more advanced MTFM probes (Galior et al., 2016; Liu et al., 2013).

\section{BIOLOGICAL APPLICATIONS OF MOLECULAR TENSION PROBES}

Molecular tension probes have been successfully used to investigate a variety of mechanosensitive biological processes in living cells (Ma and Salaita, 2019). Cell adhesion and signaling cascades which are mediated by interaction between integrin and the tripeptide motif Arg-gly-Asp (RGD), have been extensively studied by various molecular tension probes including TGT with dsDNA tether (Wang and $\mathrm{Ha}$, 2013), MTFM with PEG (Liu et al., 2013), elastic peptide (Morimatsu et al., 2013), hairpin DNA (Blakely et al., 2014; Zhang et al., 2014), and proteins (Galior et al., 2016). Due to the well-understood biological and mechanical features of integrin, integrin-RGD mechanical interaction has been routinely used to demonstrate the feasibility of newly developed molecular tension probes.

Molecular tension probes have also been used to investigate forces exerted by various other ligand-receptor interactions including the Notch receptor (Wang and $\mathrm{Ha}, 2013$ ), EGF receptor (EGFR) (Stabley et al., 2012), and T-cell receptor (TCR) (Liu et al., 2016; Ma et al., 2019). Interaction of Notch receptor with a cognate ligand activates the Notch signaling pathway, which regulates cell-cell communication and cell differentiation (Artavanis-Tsakonas et al., 1999). EGF-EGFR interaction regulates cell survival, proliferation, and differentiation (Goh et al., 2010), whereas interaction between TCR and peptide-major histocompatibility complex (pMHC) activates the adaptive immune response of T cell (Chang, 2021; Vivier and Malissen, 2005). The transmitted forces exerted by these ligand-receptor interactions were measured across the cell membrane of living cells and found to be less than $12 \mathrm{pN}$ to activate Notch receptors (Wang and $\mathrm{Ha}, 2013$ ), approximately 4 pN for EGFR (Stabley et al., 2012) and 12-19 pN for TCR (Liu et al., 2016).

\section{CONCLUSIONS AND PERSPECTIVES}

We discussed two approaches based on molecular tension probes to quantify cellular forces, TGT and MTFM. For quantification of cellular forces, TGT relies on dsDNA, which physically ruptures at a certain critical force depending on the force application geometry, whereas MTFM uses molecular springs such as polymer, hairpin DNA, and protein that extend in response to the applied force. Both techniques are highly sensitive to measure cellular forces at the $\mathrm{pN}$ level in multiple cells and can be executed by simple imaging of cells plated on the surface coated with the molecular tension probes. Both TGT and MTFM have been applied to probe the mechanics behind various mechanosensitive receptors including integrin, EGFR, and TCR.

Most molecular tension probes currently employ organic fluorophores, which tend to suffer from loss of signal due to photobleaching. Long-lasting fluorophores such as quantum dots (Cui et al., 2007) or nanodiamond (Reineck et al., 2017) would be better alternatives to design next-generation molecular tension probes. So far, molecular tension probes have been immobilized to two-dimensional (2D) flat surfaces and applied to investigate diverse mechanosensitive cellular processes in a 2D environment, which might not reflect the actual cellular response in three-dimensional (3D) environment. Application of molecular tension probes to 3D tissue culture is highly desirable to deepen our understanding of mechanotransduction in diverse mechanosensitive cellular pathways. Furthermore, super-resolved 3D imaging of molecular tension probes could provide a more detailed spatial distribution of mechanical forces exerted by single receptors during mechanotransduction.

\section{ACKNOWLEDGMENTS}

This research was supported by the National Research Foundation of Korea (NRF) funded by the Korean Government (2019R1C1C1004576) and the Chung-Ang University Research Grants in 2019.

\section{AUTHOR CONTRIBUTIONS}

K.Y.B. and H.R.K. wrote the manuscript and S.K. created the figures.

\section{CONFLICT OF INTEREST}

The authors have no potential conflicts of interest to disclose.

\section{ORCID}

Kyung Yup Baek https://orcid.org/0000-0002-8086-1171

Seohyun Kim https://orcid.org/0000-0001-7226-2470

Hye Ran Koh https://orcid.org/0000-0001-6262-7018

\section{REFERENCES}

Albrecht, C., Blank, K., Lalic-Multhaler, M., Hirler, S., Mai, T., Gilbert, I., Schiffmann, S., Bayer, T., Clausen-Schaumann, H., and Gaub, H.E. (2003). DNA: a programmable force sensor. Science 301, 367-370.

Artavanis-Tsakonas, S., Rand, M.D., and Lake, R.J. (1999). Notch signaling: cell fate control and signal integration in development. Science 284, 770776 . 
Blakely, B.L., Dumelin, C.E., Trappmann, B., McGregor, L.M., Choi, C.K., Anthony, P.C., Duesterberg, V., Baker, B.M., Block, S.M., Liu, D.R., et al. (2014). A DNA-based molecular probe for optically reporting cellular traction forces. Nat. Methods 11, 1229-1232.

Brenner, M.D., Zhou, R.B., Conway, D.E., Lanzano, L., Gratton, E., Schwartz, M.A., and $\mathrm{Ha}$, T. (2016). Spider silk peptide is a compact, linear nanospring ideal for intracellular tension sensing. Nano Lett. 16, 2096-2102.

Butcher, D.T., Alliston, T., and Weaver, V.M. (2009). A tense situation: forcing tumour progression. Nat. Rev. Cancer 9, 108-122.

Chang, J. (2021). MHC multimer: a molecular toolbox for immunologists. Mol. Cells 44, 328-334.

Clausen-Schaumann, H., Seitz, M., Krautbauer, R., and Gaub, H.E. (2000). Force spectroscopy with single bio-molecules. Curr. Opin. Chem. Biol. 4, 524-530.

Cui, B.X., Wu, C.B., Chen, L., Ramirez, A., Bearer, E.L., Li, W.P., Mobley, W.C., and $\mathrm{Chu}, \mathrm{S}$. (2007). One at a time, live tracking of NGF axonal transport using quantum dots. Proc. Natl. Acad. Sci. U. S. A. 104, 13666-13671.

Discher, D.E., Janmey, P., and Wang, Y.L. (2005). Tissue cells feel and respond to the stiffness of their substrate. Science 310, 1139-1143.

Dogterom, M. and Yurke, B. (1997). Measurement of the force-velocity relation for growing microtubules. Science 278, 856-860.

Engler, A.J., Sen, S., Sweeney, H.L., and Discher, D.E. (2006). Matrix elasticity directs stem cell lineage specification. Cell 126, 677-689.

Eroshkin, F.M. and Zaraisky, A.G. (2017). Mechano-sensitive regulation of gene expression during the embryonic development. Genesis 55, e23026.

Essevaz-Roulet, B., Bockelmann, U., and Heslot, F. (1997). Mechanical separation of the complementary strands of DNA. Proc. Natl. Acad. Sci. U. S. A. $94,11935-11940$.

Fisher, M.E. and Kolomeisky, A.B. (1999). The force exerted by a molecular motor. Proc. Natl. Acad. Sci. U. S. A. 96, 6597-6602.

Galior, K., Liu, Y., Yehl, K., Vivek, S., and Salaita, K. (2016). Titin-based nanoparticle tension sensors map high-magnitude integrin forces within focal adhesions. Nano Lett. 16, 341-348.

Gaub, B.M. and Muller, D.J. (2017). Mechanical stimulation of Piezo1 receptors depends on extracellular matrix proteins and directionality of force. Nano Lett. 17, 2064-2072.

Goh, L.K., Huang, F.T., Kim, W., Gygi, S., and Sorkin, A. (2010). Multiple mechanisms collectively regulate clathrin-mediated endocytosis of the epidermal growth factor receptor. J. Cell Biol. 189, 871-883.

Goktas, M. and Blank, K.G. (2017). Molecular force sensors: from fundamental concepts toward applications in cell biology. Adv. Mater. Interfaces 4, 1600441.

Harris, A.K., Wild, P., and Stopak, D. (1980). Silicone-rubber substrata new wrinkle in the study of cell locomotion. Science 208, 177-179.

Hirata, H., Tatsumi, H., and Sokabe, M. (2008). Mechanical forces facilitate actin polymerization at focal adhesions in a zyxin-dependent manner. $\mathrm{J}$. Cell Sci. 121, 2795-2804.

Hoffman, B.D., Grashoff, C., and Schwartz, M.A. (2011). Dynamic molecular processes mediate cellular mechanotransduction. Nature 475, 316-323.

Huse, M. (2017). Mechanical forces in the immune system. Nat. Rev. Immunol. 17, 679-690.

Jansen, K.A., Donato, D.M., Balcioglu, H.E., Schmidt, T., Danen, E.H.J., and Koenderink, G.H. (2015). A guide to mechanobiology: where biology and physics meet. Biochim. Biophys. Acta 1853(11 Pt B), 3043-3052.

Jo, M.H., Cottle, W.T., and Ha, T. (2019). Real-time measurement of molecular tension during cell adhesion and migration using multiplexed differential analysis of tension gauge tethers. ACS Biomater. Sci. Eng. 5, 3856-3863.

Jurchenko, C., Chang, Y., Narui, Y., Zhang, Y., and Salaita, K.S. (2014).
Integrin-generated forces lead to streptavidin-biotin unbinding in cellular adhesions. Biophys. J. 106, 1436-1446.

Kuo, J.C. (2013). Mechanotransduction at focal adhesions: integrating cytoskeletal mechanics in migrating cells. J. Cell. Mol. Med. 17, 704-712.

Levental, K.R., Yu, H.M., Kass, L., Lakins, J.N., Egeblad, M., Erler, J.T., Fong, S.F.T., Csiszar, K., Giaccia, A., Weninger, W., et al. (2009). Matrix crosslinking forces tumor progression by enhancing integrin signaling. Cell 139, 891906.

Li, H.Y., Zhang, C., Hu, Y.R., Liu, P.X., Sun, F., Chen, W., Zhang, X.H., Ma, J., Wang, W.X., Wang, L., et al. (2021). A reversible shearing DNA probe for visualizing mechanically strong receptors in living cells. Nat. Cell Biol. 23, 642-651.

Liu, Y., Blanchfield, L., Ma, V.P.Y., Andargachew, R., Galior, K., Liu, Z., Evavold, B., and Salaita, K. (2016). DNA-based nanoparticle tension sensors reveal that $\mathrm{T}$-cell receptors transmit defined $\mathrm{pN}$ forces to their antigens for enhanced fidelity. Proc. Natl. Acad. Sci. U. S. A. 113, 56105615.

Liu, Y., Galior, K., Ma, V.P.Y., and Salaita, K. (2017). Molecular tension probes for imaging forces at the cell surface. Acc. Chem. Res. 50, 29152924.

Liu, Y., Yehl, K., Narui, Y., and Salaita, K. (2013). Tension sensing nanoparticles for mechano-imaging at the living/nonliving interface. J. Am. Chem. Soc. 135, 5320-5323.

Ma, R., Kellner, A.V., Ma, V.P.Y., Su, H.Q., Deal, B.R., Brockman, J.M., and Salaita, K. (2019). DNA probes that store mechanical information reveal transient piconewton forces applied by T cells. Proc. Natl. Acad. Sci. U. S. A. 116, 16949-16954.

Ma, V.P.Y., Liu, Y., Yehl, K., Galior, K., Zhang, Y., and Salaita, K. (2016). Mechanically induced catalytic amplification reaction for readout of receptor-mediated cellular forces. Angew. Chem. Int. Ed. Engl. 55, 54885492.

Ma, V.P.Y. and Salaita, K. (2019). DNA nanotechnology as an emerging tool to study mechanotransduction in living systems. Small 15, e1900961.

Mammoto, A., Mammoto, T., and Ingber, D.E. (2012). Mechanosensitive mechanisms in transcriptional regulation. J. Cell Sci. 125, 3061-3073.

Martinac, B. (2004). Mechanosensitive ion channels: molecules of mechanotransduction. J. Cell Sci. 117, 2449-2460.

Morimatsu, M., Mekhdjian, A.H., Adhikari, A.S., and Dunn, A.R. (2013). Molecular tension sensors report forces generated by single integrin molecules in living cells. Nano Lett. 13, 3985-3989.

Murad, Y. and Li, I.T.S. (2019). Quantifying molecular forces with serially connected force sensors. Biophys. J. 116, 1282-1291.

Neuman, K.C. and Nagy, A. (2008). Single-molecule force spectroscopy: optical tweezers, magnetic tweezers and atomic force microscopy. Nat. Methods 5, 491-505.

Parsons, J.T., Horwitz, A.R., and Schwartz, M.A. (2010). Cell adhesion: integrating cytoskeletal dynamics and cellular tension. Nat. Rev. Mol. Cell Biol. 11, 633-643.

Pramanik, A. (2004). Ligand-receptor interactions in live cells by fluorescence correlation spectroscopy. Curr. Pharm. Biotechnol. 5, 205212.

Reineck, P., Capelli, M., Lau, D.W.M., Jeske, J., Field, M.R., Ohshima, T., Greentree, A.D., and Gibson, B.C. (2017). Bright and photostable nitrogenvacancy fluorescence from unprocessed detonation nanodiamond. Nanoscale 9, 497-502.

Riesenberg, C., Iriarte-Valdez, C.A., Becker, A., Dienerowitz, M., Heisterkamp, A., Ngezahayo, A., and Torres-Mapa, M.L. (2020). Probing ligand-receptor interaction in living cells using force measurements with optical tweezers. Front. Bioeng. Biotechnol. 8, 598459.

Roca-Cusachs, P., Conte, V., and Trepat, X. (2017). Quantifying forces in cell biology. Nat. Cell Biol. 19, 742-751. 
Roy, P., Rajfur, Z., Pomorski, P., and Jacobson, K. (2002). Microscope-based techniques to study cell adhesion and migration. Nat. Cell Biol. 4, E91-E96.

Stabley, D.R., Jurchenko, C., Marshall, S.S., and Salaita, K.S. (2012). Visualizing mechanical tension across membrane receptors with a fluorescent sensor. Nat. Methods 9, 64-67.

Style, R.W., Boltyanskiy, R., German, G.K., Hyland, C., MacMinn, C.W., Mertz, A.F., Wilen, L.A., Xu, Y., and Dufresne, E.R. (2014). Traction force microscopy in physics and biology. Soft Matter 10, 4047-4055.

Uroz, M., Wistorf, S., Serra-Picamal, X., Conte, V., Sales-Pardo, M., RocaCusachs, P., Guimera, R., and Trepat, X. (2018). Regulation of cell cycle progression by cell-cell and cell-matrix forces. Nat. Cell Biol. 20, 646-654.

Vining, K.H. and Mooney, D.J. (2017). Mechanical forces direct stem cell behaviour in development and regeneration. Nat. Rev. Mol. Cell Biol. 18, 728-742.

Vivier, E. and Malissen, B. (2005). Innate and adaptive immunity: specificities and signaling hierarchies revisited. Nat. Immunol. 6, 17-21.

Vogel, V. and Sheetz, M. (2006). Local force and geometry sensing regulate cell functions. Nat. Rev. Mol. Cell Biol. 7, 265-275.

Wang, N., Butler, J.P., and Ingber, D.E. (1993). Mechanotransduction across the cell-surface and through the cytoskeleton. Science 260, 1124-1127.
Wang, X.F. and Ha, T. (2013). Defining single molecular forces required to activate integrin and notch signaling. Science 340, 991-994.

Wang, X.F., Sun, J., Xu, Q., Chowdhury, F., Roein-Peikar, M., Wang, Y.X., and $\mathrm{Ha}$, T. (2015). Integrin molecular tension within motile focal adhesions. Biophys. J. 109, 2259-2267.

Wang, Y.L., LeVine, D.N., Gannon, M., Zhao, Y.C., Sarkar, A., Hoch, B., and Wang, X.F. (2018). Force-activatable biosensor enables single platelet force mapping directly by fluorescence imaging. Biosens. Bioelectron. $100,192-200$.

Wang, Y.X., Chang, J., Chen, K.D., Li, S., Li, J.Y.S., Wu, C.Y., and Chien, S. (2007). Selective adapter recruitment and differential signaling networks by VEGF vs. shear stress. Proc. Natl. Acad. Sci. U. S. A. 104, 8875-8879.

Woodside, M.T., Behnke-Parks, W.M., Larizadeh, K., Travers, K., Herschlag, D., and Block, S.M. (2006). Nanomechanical measurements of the sequence-dependent folding landscapes of single nucleic acid hairpins. Proc. Natl. Acad. Sci. U. S. A. 103, 6190-6195.

Zhang, Y., Ge, C.H., Zhu, C., and Salaita, K. (2014). DNA-based digital tension probes reveal integrin forces during early cell adhesion. Nat. Commun. 5, 5167. 